\title{
Magnetic turbulence and particle dynamics in the Earth's magnetotail
}

\author{
G. Zimbardo ${ }^{1, *}$, A. Greco ${ }^{1, *}$, A. L. Taktakishvili ${ }^{2}$, P. Veltri ${ }^{1, *}$, and L. M. Zelenyi ${ }^{3}$ \\ ${ }^{1}$ Physics Department, University of Calabria, Ponte P. Bucci, Cubo 31C, I-87036 Rende, Italy \\ ${ }^{2}$ Abastumani Astrophysical Observatory, A. Kazbegi str., 2a, 380060, Tbilisi, Georgia \\ ${ }^{3}$ Space Research Institute, Profsoyuznaya 84/32, 117810 Moscow, Russia \\ *also at: Istituto Nazionale di Fisica della Materia, Unita' di Cosenza, Italy
}

Received: 3 October 2002 - Revised: 14 March 2003 - Accepted: 19 March 2003

\begin{abstract}
The influence of magnetic turbulence in the nearEarth magnetotail on ion motion is investigated by numerical simulation. The magnetotail current sheet is modelled as a magnetic field reversal with a normal magnetic field component $B_{n}$, plus a three-dimensional spectrum of magnetic fluctuations $\delta \mathbf{B}$ which represents the observed magnetic turbulence. The dawn-dusk electric field $E_{y}$ is also considered. A test particle simulation is performed using different values of $B_{n}$ and of the fluctuation level $\delta B / B_{0}$. We show that when the magnetic fluctuations are taken into account, the particle dynamics is deeply affected, giving rise to an increase in the cross tail transport, ion heating, and current sheet thickness. For strong enough turbulence, the current splits in two layers, in agreement with recent Cluster observations.
\end{abstract}

Key words. Magnetospheric physics (magnetospheric configuration and dynamics) - Interplanetary physics (MHD waves and turbulence) - Electromagnetics (numerical methods)

\section{Introduction}

The Earth's magnetotail is a large reservoir of plasma and energy, and is characterized by a magnetic configuration which leads to many intriguing nonlinear phenomena. In particular, several spacecraft have observed phenomena as sudden plasma motions, current diversions, energetic particle bursts, and strong geomagnetic activity mixed with relatively quiet periods. The observations of ISEE 2, AMPTE/IRM, Geotail, Interball, and other spacecraft have shown the existence of plasma and magnetic field turbulence in the distant and near Earth's magnetotail (Angelopoulos et al., 1993; Hoshino et al., 1994; Ohtani et al., 1995; Bauer et al., 1995; Borovsky et al., 1997). Usually, the turbulence is stronger in the center of the plasma sheet (Bauer et al., 1995; Hoshino et al., 1996), while a more regular magnetic configuration is found in the vicinity of the magnetospheric lobes. The observed

Correspondence to: G. Zimbardo (zimbardo@ fis.unical.it) fluctuations have a power law spectrum which indicates the presence of the nonlinear interactions typical of turbulence (Malara et al., 1992; Bauer et al., 1995; Ohtani et al., 1995; Hoshino et al., 1994; Borovsky et al., 1997). Although the magnetic turbulence is stronger during active geomagnetic periods, it is non-negligible even during the quiet times.

Analytical studies and self-consistent numerical simulations, both fluid and kinetic (Lui et al., 1991; Malara et al., 1992; Pritchett and Büchner, 1995; Daughton, 1998, 1999; Wiegelmann and Büchner, 2000; Peroomian et al., 2000; Lapenta and Brackbill, 2000), were developed to study the growth of current sheet instabilities. The structure of the magnetotail current sheet also was studied by hybrid simulations which treat the electrons as a charge neutralizing fluid (Hesse et al., 1995, 1996). Further information, like cause and effect relationships, can be obtained by test particle simulations which take into account the two-dimensional (2-D) and three-dimensional (3-D) geometry of the magnetosphere (Ashour-Abdalla et al., 1994, 1996; Delcourt and Belmont, 1998), the particle acceleration in self-consistent 2-D turbulence (Matthaeus et al., 1984), the electric field morphology (Antonova and Ovchinnikov, 1999), and the presence of magnetic turbulence in the distant (Veltri et al., 1998) and in the near Earth (Greco et al., 2002) magnetotail.

In a previous study, Veltri et al. (1998) considered the distant magnetotail, where the average component of the magnetic field normal to the current sheet $B_{n}$ is statistically negligible, and found for fluctuation levels $\delta B / B_{0}>\sim 0.2$ that the current sheet has a double layer structure, with a current density minimum in the center of the quasi-neutral sheet. Also, Veltri et al. (1998) found that the fluctuations enhance ion heating and contribute to the formation of the equilibrium structure. Recently, Greco et al. (2002) considered the near-Earth magnetotail, where $B_{n}>0$, and found that even in this case the magnetic fluctuations induce a double layer structure in the current sheet, although a stronger $\delta B / B_{0}$ is usually required to achive this effect. In this paper we investigate ion dynamics in the presence of magnetic turbulence in the near Earth and middle magnetotail, where $B_{n}$ is north- 
ward oriented. The considered configuration can be a (local) magnetotail model for the growth phase of magnetospheric substorms. With respect to Greco et al. (2002), we extend the turbulence spectrum by including in the model a number of long wavelength modes, which may correspond to the formation of large magnetic islands. We study the influence of $B_{n}$ on the distribution function moments, and we find that it effectively controls the particle dynamics. When including magnetic turbulence, the vertical current profile thickens and, for sufficient fluctuation levels $\delta B / B_{0}$, the current splits into two layers. Also, ion heating and isotropization is considerably enhanced. In practice, the magnetic fluctuations play the role of an effective scattering mechanism, but also allow easier motion along $y$ at the edges of the current sheet.

In Sect. 2 we set up the model magnetic field and we describe the test particle simulation. In Sect. 3 we present the simulation results, and we discuss them in connection with the observations. In Sect. 4 we give the conclusions.

\section{Numerical model}

The considered magnetic field configuration consists of an unperturbed, sign reversing component, directed along the Earth-Sun axis, $\mathbf{B}_{0 x}(z)=B_{0 x}(z) \mathbf{e}_{x}$, such that $B_{0 x}(z)=$ $-B_{0 x}(-z)$; an unperturbed, constant, normal component $\mathbf{B}_{n}=B_{n} \mathbf{e}_{z}$; and of 3-D stationary magnetic fluctuations $\delta \mathbf{B}(\mathbf{r})$ having a power law spectrum (see below). Thus, the total magnetic field is given by $\mathbf{B}=\mathbf{B}_{0 x}(z)+\mathbf{B}_{n}+\delta \mathbf{B}(\mathbf{r})$. Following Veltri et al. (1998), we use for $B_{0 x}(z)$ the expression of a modified Harris magnetic field reversal,

$$
B_{0 x}(z)=B_{0} \frac{\tanh (z / \lambda)-(z / \lambda) \cosh ^{-2}(L / 2 \lambda)}{\tanh (L / 2 \lambda)-(L / 2 \lambda) \cosh ^{-2}(L / 2 \lambda)} .
$$

Here, $L$ is the total thickness of the considered magnetic field configuration (i.e. of the simulation box) and $\lambda=0.25 L$ is the current sheet half thickness. The unperturbed components describe the parabolic field line geometry which is found in the Earth's tail from, approximately, 10 to $60 R_{E}$ downtail.

The power law spectrum of the magnetic fluctuations, regularly observed by the Geotail (Hoshino et al., 1994), AMPTE/IRM (Bauer et al., 1995), ISEE 2 (Borovsky et al., 1997) and Interball satellites (Zelenyi et al., 1998) in this region of the magnetotail, has well-defined, "reproducible" features like the spectral shape, the frequency range, and the fluctuation level for given geomagnetic activity levels. This suggests that magnetic turbulence is in a steady state, in a statistical sense. We represent these fluctuations as the sum of static magnetic perturbations (Zimbardo et al., 1995; Veltri et al., 1998; Greco et al., 2002)

$\delta \mathbf{B}(\mathbf{r})=\sum_{k, \sigma} \delta B(\mathbf{k}) \mathbf{e}_{\sigma}(\mathbf{k}) \exp \left(i\left(\mathbf{k} \cdot \mathbf{r}+\phi_{\mathbf{k}}^{\sigma}\right)\right)$,

where

$\delta B(\mathbf{k})=\frac{C}{\left(k_{x}{ }^{2} l_{x}^{2}+k_{y}{ }^{2} l_{y}{ }^{2}+k_{z}{ }^{2} l_{z}^{2}+1\right)^{\alpha / 4+1 / 2}}$ and where $\mathbf{e}_{\sigma}(\mathbf{k})$ are the polarization unit vectors (Veltri et al., 1998), $C$ is a normalization constant, and $l_{x}, l_{y}, l_{z}$ are the turbulence correlation lengths in the $x, y, z$ directions, respectively. While for the distant magnetotail Veltri et al. (1998) assumed as the spectral index $\alpha=1.5$ (even though a large variability of this index is often observed, Hoshino et al., 1994), for the near-Earth magnetotail $\alpha=2.3$ may be appropriate (Slavin et al., 1985; Ohtani et al., 1995; Borovsky et al., 1997; Zelenyi et al., 1998). Anyway, we anticipate that the influence of the spectral index on the simulation results is rather small.

For the runs presented here, the wave-vector components are chosen on a grid such that $k_{i}=2 \pi n_{i} / l_{i}, i=x, y, z$, where $n_{i}$ are integers satisfying $\left(n_{x}^{2}+n_{y}^{2}+n_{z}^{2}\right)<144$. In Greco et al. (2002) we also imposed $\left(n_{x}^{2}+n_{y}^{2}\right)>6$, in order to avoid the dominance of long wavelength modes, which have the largest amplitudes. Here, however, we keep the long wavelength modes, too, in order to mimic the presence of magnetic islands or other coherent structures with scales of a few Earth radii (Runov et al., 2003). Note that the longest modes present in $\delta \mathbf{B}$ have a wavelength $\lambda=5 \mathrm{~L}$. In addition to the above magnetic field configuration, we assume that there is a constant cross tail electric field, oriented in the dawn to dusk direction, $\mathbf{E}=E_{y} \mathbf{e}_{y}$ (see Greco et al., 2002; Veltri et al., 1998, for more details on the magnetic turbulence model). Hereinafter, $\delta B=\sqrt{\langle\delta \mathbf{B} \cdot \delta \mathbf{B}\rangle}$, with the average made over the simulation box. We also define $\delta b \equiv \delta B / B_{0}$ and $b_{n} \equiv B_{n} / B_{0}$.

In this paper we consider the electrons as a charge neutralizing background, and leave a more detailed study of their influence for future work. Although we are considering static magnetic perturbations, it can be shown that the fluctuating electric field (produced by realistic magnetic perturbations) has a negligible impact on particle dynamics (Greco et al., 2002). The equations of motion can be written in a dimensionless form by normalizing all length scales to the unit length $L$, velocities to electric drift velocity $V_{E}=c E_{y} / B_{0}$, magnetic and electric fields to $B_{0}$ and $E_{y}$, respectively, and time to ion gyrofrequency $\omega_{0}=e B_{0} / m_{i} c$. Typical values of the normalization parameters for the near-Earth magnetotail can be obtained by setting $B_{0}=10-20 \mathrm{nT}, B_{n}=1-2 \mathrm{nT}$, and $E_{y}=0.1 \mathrm{mV} / \mathrm{m}$. The unit length may be assumed to be $L=10^{4} \mathrm{~km}$, which implies a current sheet thickness $\lambda=0.25 L=2500 \mathrm{~km}$.

The particles are injected at the upper and lower boundary of the simulation box, at $z= \pm 0.5 \mathrm{~L}$, with random positions in the $x y$ plane, and with velocities distributed according to a shifted Maxwellian (Ashour-Abdalla et al., 1994)

$f\left(v_{\|}, v_{\perp}\right)=\left(\sqrt{2 \pi} v_{t h}^{3}\right)^{-1} \exp \left(-\frac{\left(v_{\|}-u\right)^{2}+v_{\perp}^{2}}{2 v_{t h}^{2}}\right)$.

Here, $u$ is the streaming velocity and $v_{t h}$ is the thermal velocity. Following Ashour-Abdalla et al. (1994), we assume that $u=200 \mathrm{~km} / \mathrm{s}$ and $v_{t h}=170 \mathrm{~km} / \mathrm{s}$, corresponding to a particle energy of $0.3 \mathrm{keV}$ (see Greco et al., 2002, for a full description of the injection routine). 


\section{Numerical results}

In the present simulations we assume a value of the normal magnetic field of $b_{n}=0.05$, which is typical of the magnetotail at pre-onset (Petrukovich, 2000). The corresponding value of the stochasticity parameter $\kappa_{i}$ (Büchner and Zelenyi, $1986,1989)$ is $\kappa_{i} \simeq 0.21$, so that ion motion in the quasineutral sheet is not adiabatic, but is in the quasi-adiabatic regime characterized by $\kappa_{i}<1$. This means that the particles can have meandering orbits in the quasi-neutral sheet. At injection, the ion Larmor radius in the asymptotic field $B_{0}$ is $\rho_{0} \simeq 0.026 L$, so that $\rho_{0} \simeq \lambda / 10$. Thus, our parameters correspond to a moderately thick current sheet (e.g. Wiegelmann and Büchner, 2000). Sample particle trajectories can be found in Zimbardo et al. (2000) and Greco et al. (2002).

In order to gain an understanding of the typical ion dynamics, we inject many particles in the simulation box and numerically integrate their equation of motion. Then we compute the distribution function moments as density $n$, bulk velocity $\mathbf{V}$, current density $\mathbf{j}$, temperature $T$, etc., on a threedimensional grid with 20 grid points in $x, 400$ in $y$, and 40 in $z$. Typically, 50000 particles are injected for each run. In a test particle simulation, the normalization for the number density is, to a good extent, arbitrary. Here, it is based on the consideration that the ion current $I_{y}$ has to be strong enough to reproduce the unperturbed magnetic field $B_{0 x}(z)$ introduced in our model. Indeed, Ampere's law leads to $2 B_{0} L_{x}=4 \pi I_{y} / c$, with $I_{y}=\int j_{y} d x d z$ the total current across a section of the current sheet of length $L_{x}$. On the other hand, velocity is expressed in units of $V_{E}$ (see Sect. 2), so that the normalization for density is obtained as $n^{*}=I_{y} /\left[e \int V_{y} d x d z\right]$, with $I_{y}$ constant for all the runs (this implies that when the average value of $V_{y}$ is large, the density is low).

In Fig. 1 we show the two-dimensional contours, from top to bottom, of $n, V_{x}, V_{y}, V_{z}$, the $y$ component of the current density $j_{y}$, and of the parallel and perpendicular temperatures, $T_{\|}=\left(m_{i} / n k_{B}\right) \int\left(v_{\|}-V_{\|}\right)^{2} f d^{3} v$ and $T_{\perp}=\frac{1}{2}\left(T-T_{\|}\right)$, for $\delta b=0, b_{n}=0.05$, and $E_{y}=0.1 \mathrm{mV} / \mathrm{m}$. Here, $k_{B}$ is Boltzmann's constant. For each panel, we have $-10 L<$ $y<10 L$ and $-0.5 L<z<0.5 L$, corresponding to the whole simulation box, while an average over $x$ was performed. In all panels of Figures 1 and 2, strong variations at the left boundary $(y=-10 L)$ can be seen: these are an artifact of the injection scheme (the injection zone corresponds to the range $-10 L<y<10 L$ ) and are due to the fact that the cross tail electric field pushes the particles toward positive $y$. Since there are no particles coming from $y<-10 \mathrm{~L}$, a particle depletion is found close to the left boundary. In the top panel the plasma number density is reported. The density appears to be larger at the $z$-boundaries of the simulation box, because the injected particles are mirrored toward the center where $|\mathbf{B}|$ is smaller, so that, in the absence of any scattering agent, particles are faster in the center and give a reduced contribution to number density.

In Fig. 1 it can be seen that the normal component $B_{n}$ has a strong influence in organizing the particle motion. In par- ticular, the motion along $y$, the cross tail direction, is inhibited by $B_{n}$ in spite of the presence of the electric field $E_{y}$, except for the central layer where ion motion is unmagnetized. The $x$ component of the bulk velocity, second panel, is strongly negative. Taking into account the fact that the $x$ axis is oriented toward the tail, this means that the plasma flow is toward the Earth, with the well-known earthward convection due to the dawn-dusk electric field. On the other hand, $V_{y}$ is oriented along the electric field, and is clearly larger in the central band around $z=0$, where $\left|B_{0 x}\right|$ is smaller and the ions are unmagnetized. Some negative values of $V_{y}$ (and of $j_{y}$ ), corresponding to the dark blue areas, can also be seen. These diamagnetic current wings are due to a combination of drift effects (Zelenyi et al., 2000). The $z$ component of velocity has smaller values than the other two components, is zero on average, and there are no spatial features. This means that at any location in the simulation box there are as many particles going up along $z$ as there are going down. The current density $j_{y}$ has features similar to $V_{y}$. The lowest two panels show the temperatures $T_{\|}$and $T_{\perp}$, computed with respect to the local magnetic field. We can see that $T_{\perp}$ is very nearly the same as at injection, since very small acceleration by $E_{y}$ is allowed. However, $T_{\|}$appears to be somewhat larger than $T_{\perp}$, since the streaming velocity $u$ given at injection gives rise to two counterstreaming beams which increase the spread in parallel velocity and hence $T_{\|}$.

When magnetic turbulence is included in the simulation, the results change in a significant way. In Fig. 2 we show the same quantities of Fig. 1, for the same value of $b_{n}=0.05$, while $\delta b=0.5$. In order to ease the comparison between the two figures, the same color scales are used in both figures (that is why some panels in Fig. 1 do not show many colors), except for the density. In Fig. 2 it can be seen that the density is somewhat peaked at the center of the neutral sheet, that the current carrying region is considerably inflated, and that cross tail motion away from $z \simeq 0$ is no longer inhibited. We can see that the magnetic fluctuations scramble the oscillatory, bouncing $z$-motion of the injected particles which creates, for $\delta b=0$, a number density depression in the central region. As a consequence, in the presence of turbulence particles spend more time in the central region, giving rise to a more physical density profile. The influence of long wavelength modes, with periodicity $\lambda=5 \mathrm{~L}$, can be seen in the density plot as a series of density enhancements, which we argue are located where the fluctuating magnetic field is weaker, as a result of the random phases in (2). The density plot shows that strong magnetic turbulence, with $\delta B / B_{0} \simeq 0.5$, can generate a factor 2 in density variations. Also, the modulus of the velocity $V_{x}$ is decreased in the central region, since the magnetic fluctuations disturb the earthward convection: indeed, $\delta b$ is larger than $b_{n}$, so that locally the $\boldsymbol{E} \times \boldsymbol{B}$ drift can be in different directions than $x$. On average, the drift in the $x$ direction prevails but the changing direction of motion slows down the earthward drift. The influence of magnetic turbulence on the velocity $V_{y}$ is twofold: on the one hand, turbulence causes the peak of the velocity to decrease because it scatters the ions around. On the other 


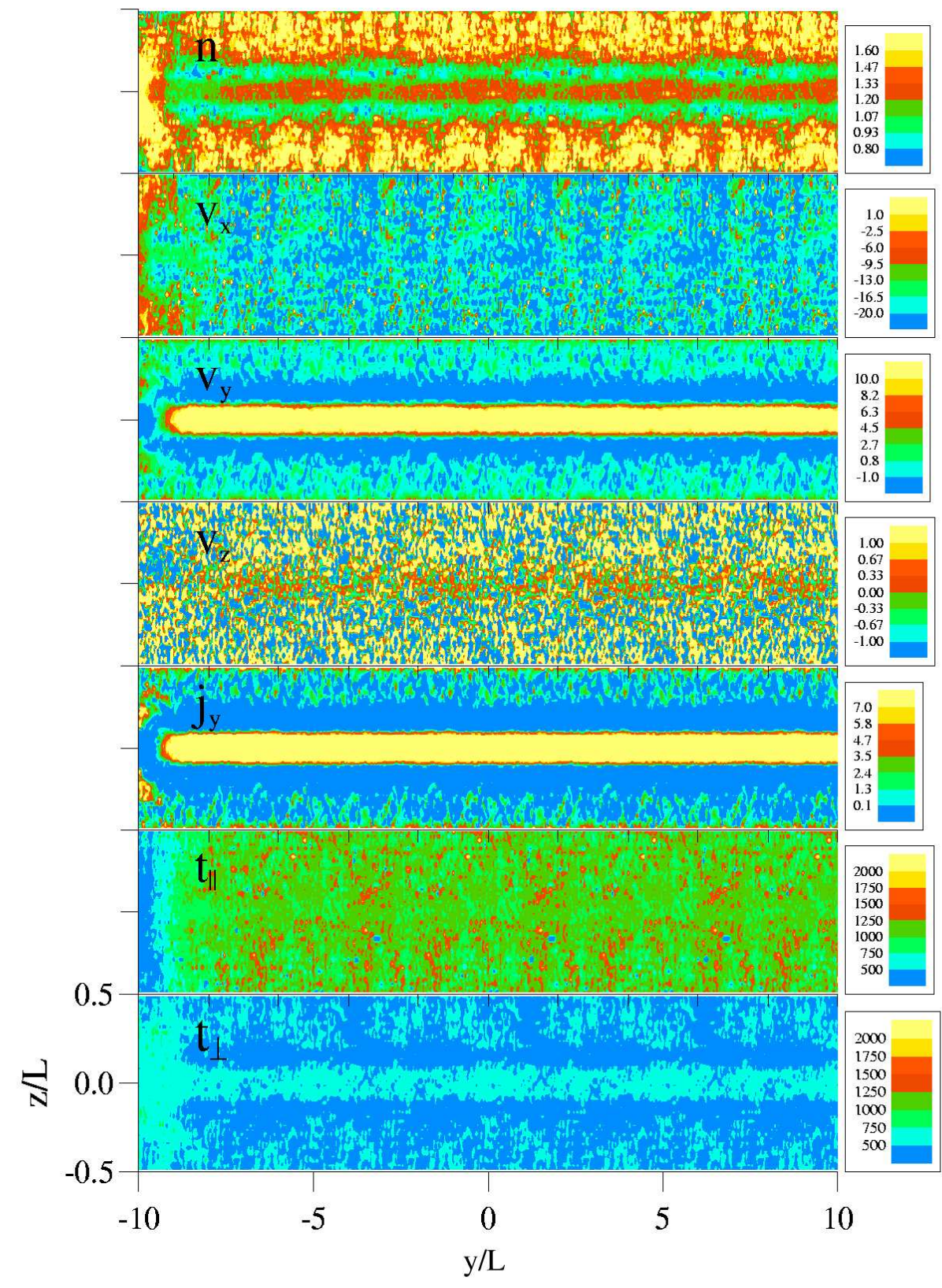

Fig. 1. Contour plots of the ion moments. From top to bottom, the density $n$, the three components of the bulk velocity $V_{x}, V_{y}$, and $V_{z}$, the current density $j_{y}$, the temperatures $T_{\|}$and $T_{\perp}$. For this run $\delta b=0$ and $b_{n}=0.05$. Density is in units of $n^{*}$, velocity in units of $V_{E}$, currents density in units of $c B_{0} / 4 \pi L$, and temperatures in units of $m_{i} V_{E}^{2} / k_{B}$. Color scales on the right. hand, the velocity for $|z|>0.1 L$ increases because of the presence of particles scattered away from the center, and because the fluctuations $\delta B_{y}$ bend the field lines also in the $y$ direction. This allows cross field motion (with respect to the average field) even for magnetized particles, although of a biased diffusive type, since $\left\langle\delta B_{y}\right\rangle=0$ and $E_{y} \neq 0$. However, when $\delta b$ becomes large, the magnetic turbulence also slows down the ion motion because ions are strongly scattered (Veltri et al., 1998).

The temperature is greatly increased with respect to Fig. 1, showing that the magnetic turbulence scrambles the ordered velocity gained because of $E_{y}$ into all directions, leading to an effective thermalization of the potential drop. Also, $T_{\|}$and $T_{\perp}$ are larger in the center of the simulation box and grow to- wards the right, where energization from the electric field is favoured. Greco et al. (2002) have shown that ion heating progressively increases with the turbulence level, although there is no critical turbulence level above which heating appears. The ion heating is strong since the ion temperature reaches more than 3 times the injection temperature. Once the numerical values are set into the estimates of the ion thermal energy density and of the turbulence energy density, they turn out to be of the same order of magnitude. However, ion heating comes from the dawn-dusk potential drop, not from the dissipation of magnetic turbulence, which is not considered in our simulation. The nonlinear feedback of ion motion and heating on the magnetic turbulence will be considered in a future work. Note that there are only minor differences be- 


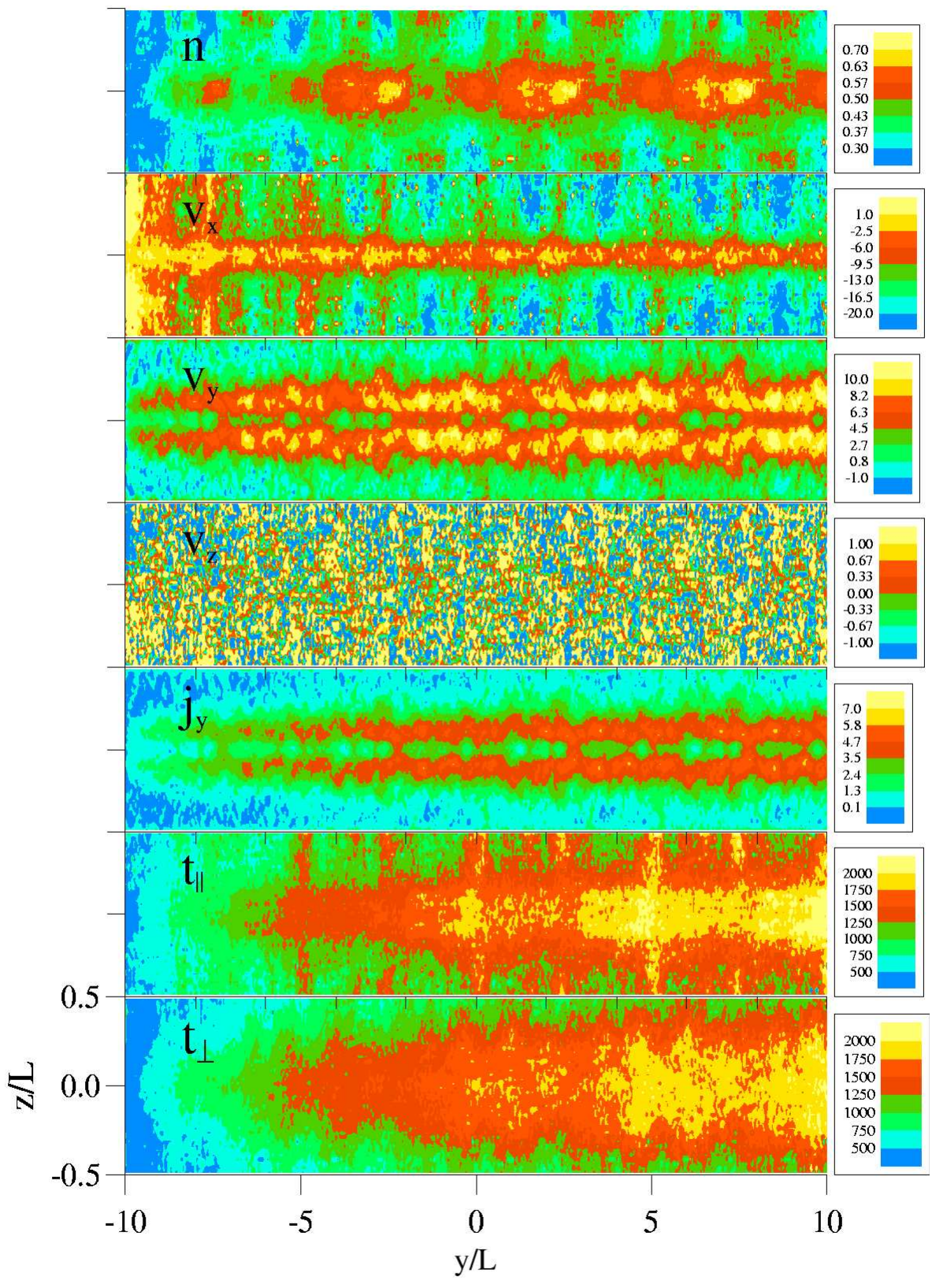

Fig. 2. Same as Fig. 1 but for $\delta b=0.5$ and $b_{n}=0.05$. tween $T_{\|}$and $T_{\perp}$, showing that heating is nearly isotropic. The comparison between Figs. 1 and 2 shows that the magnetic fluctuations lead to a fast pitch angle diffusion, which results in isotropizing the temperature.

Both $j_{y}$ and $V_{y}$ exhibit a marked double layer structure. This structure, which is also inferred from the observations (Sergeev et al., 1993; Hoshino et al., 1996), is due in our numerical model to the enhanced braking at $z \simeq 0$, due to the concentration of turbulence in the quasi-neutral sheet, which models the observations (Bauer et al., 1995). A splitting in a double humped current profile was also obtained by AshourAbdalla et al. (1994) and Delcourt and Belmont (1998), by using a three-dimensional magnetic configuration at large scales but without magnetic turbulence; in those cases, the current splitting was due to the ion meandering orbits which imply maximum (and positive) $v_{y}$ away from $z=0$. However, in our model, enhanced pitch angle scattering masks the effect of meandering orbits, and no current splitting is obtained without magnetic fluctuations. Therefore, the physical origin of the current splitting reported here lies in the disturbance to ion motion due to magnetic turbulence (Sergeev et al., 1993). It is noteworthy that recent magnetic field observations by the Cluster mission unambiguously showed that the magnetotail current sheet at about $20 R_{E}$ downtail can exhibit a bifurcated structure (Nakamura et al., 2002; Runov et al., 2003; Sergeev et al., 2003). These bifurcated current sheets appear to not necessarily be associated with the substorm expansion phase, that is with dipolarization of fast 
flows. It would be interesting to assess the magnetic fluctuation level during those events in order to see whether the bifurcated structure can be attributed to the influence of magnetic turbulence, as reported here.

We have computed the $z$ profiles (averaged over $x$ and $y$ ) of $n, V_{y}, j_{y}$ and of the other distribution function moments for several values of $\delta b$ and of $b_{n}$ (Greco et al., 2002). The main results of such a parametric study can be summarized as follows. It appears that the presence of magnetic turbulence has a strong influence on the global ion dynamics, inflating the current carrying region and favouring the thermalization of the kinetic energy gained by ions in the electric potential drop, as shown in Fig. 2. A substantial level of turbulence, $\delta B / B_{0}>0.3$ is needed to attain pressure balance normal to the current sheet. From this point of view, magnetic turbulence appears to be a basic ingredient of the magnetotail equilibrium structure, both for the distant (Veltri et al., 1998) and for the near earth tail (Greco et al., 2002). In many respects, $B_{n}$ and $\delta B$ have opposite effects on the current structure and ion heating. Indeed, a large value of $B_{n}$ inhibits the $y$ motion in the quasi-neutral sheet, while magnetic fluctuations favor $y$ motion at some distance of the center; the strong magnetic turbulence in the center of the current sheet slows down the ion motion and causes the double humped profiles of the current and of ion velocity. The level of $\delta B / B_{0}$ needed for current splitting grows with that of $B_{n}$ : we argue that this is due to the fact that for large $B_{n}$ the ions cross the sheet rather quickly, with the normal component acting like a guide field. Indeed, the stochasticity paramenter $\kappa_{i}$ grows with $B_{n}$ and for $\kappa_{i} \rightarrow 1$ the length of the meandering orbits is strongly reduced; as a consequence, the ions have less time to interact with the magnetic turbulence and to feel the braking on $V_{y}$ in the center of the current sheet.

When the ion simulation is run with the average magnetic field profile corresponding to the computed current, current splitting persists, so that it can be considered a self-consistent feature of the current sheet (Greco et al., 2002). For low $\delta B$ and moderate anisotropy of the incoming distribution function, negative diamagnetic current wings are found in the current profile, as shown in Fig. 1, with corresponding overshoots in the magnetic field profile, a feature which is sometimes observed (e.g. Sergeev et al., 2003). Thus, our model helps to understand the influence of magnetic fluctuations and of the normal component on the bulk features of the ions in the magnetotail, and allows one to explain some experimental features. The current splitting may be considered as the beginning of the current diversion process at the onset of magnetospheric substorms, when $B_{n}$ decreases and $\delta B$ usually increases, while ion heating grows with $\delta B$ and decreases with $B_{n}$, in agreement with the observations (Petrukovich, 2000).

\section{Conclusions}

We studied the ion dynamics in the magnetotail in the presence of a stationary magnetic turbulence $\delta B$ and of the nor- mal component of the magnetic field $B_{n}$. This study is relevant to the substorm growth and early expansion phases of the magnetotail dynamics when, in the tail thinning phase, $B_{n}$ becomes smaller while the tail stretches in the antisolar direction. The magnetic turbulence observed by several spacecraft was modeled numerically, and a test particle simulation was performed in which ions are injected into the quasi-neutral current sheet. The fluctuation level $\delta B / B_{0}$ and the normal component $B_{n}$ were given values relevant to the near-Earth magnetotail. The presence of long wavelength modes causes inhomogeneities in the plasma density, as is sometimes observed. For a substantial level of fluctuations, $\delta B / B_{0}>0.2$, the current splits into two sheets for $B_{n} \sim 0$ (Veltri et al., 1998), but increasing $B_{n}$ requires a higher $\delta B / B_{0}$ in order to have the current splitting (Greco et al., 2002). Also, ion heating increases with the increase in $\delta B / B_{0}$ and with the decrease in $B_{n}$, as these changes favor motion perpendicular to the average magnetic field and along the potential drop. The magnetic fluctuations are very effective in thermalizing the directed kinetic energy gained from the electric potential drop, in causing pitch angle scattering, and in isotropizing the ion temperature. Our results agree well with previous (Sergeev et al., 1993; Hoshino et al., 1996) and recent (Nakamura et al., 2002; Runov et al., 2003; Sergeev et al., 2003) spacecraft observations of bifurcated current sheets and allows one to interpret them as the effect of magnetic turbulence. Further study is needed to assess the electron contribution to the magnetotail current structure in the presence of turbulence. Altogether, it appears that magnetic turbulence is not only the product of plasma instabilities and nonlinear dynamics, but also an active physical phenomenon that can help to set up both the magnetotail equilibrium structure and the scenario of magnetospheric substorms evolution.

Acknowledgements. It is a pleasure to thank A. V. Milovanov and A. A. Petrukovich for fruitful discussions. This work was supported by the Italian MIUR through a National Project Fund (Cofin 2000) and by the European Union with the Research Training Network "Turbulence in Space Plasmas: Theory, Observations, and Simulations". Numerical simulations were performed in the framework of the HPCC (Center for High Performance Computing) at the University of Calabria. L. M. Z. acknowledges the support of RFFI grant 01-02-16637 and of the Humboldt foundation in his theoretical studies. During his stays in Italy A. L. T. was supported by grants of Italian INFM and MUIR.

Topical Editor T. Pulkkinen thanks two referees for their help in evaluating this paper.

\section{References}

Angelopoulos, V., Kennel, C. F., Coroniti, F. V., et al.: Characteristics of ion flow in the quiet state of the inner plasma sheet, Geophys. Res. Lett., 20, 1711, 1993.

Antonova, E. E. and Ovchinnikov, I. L.: Magnetostatically equilibrated plasma sheet with developed medium scale turbulence: structure and implications for substorm dynamics, J. Geophys. Res., 104, 17 289, 1999. 
Ashour-Abdalla, M., Zelenyi, L. M., Peroomian, V., and Richard, R.: Consequences of magnetotail ion dynamics, J. Geophys. Res., 99, 14 891, 1994.

Ashour-Abdalla, M., Frank, L. A., Paterson, W. R., Peroomian, V., and Zelenyi, L. M.: Proton velocity distributions in the magnetotail: theory and observations, J. Geophys. Res., 101, 2587, 1996.

Bauer, T. M., Baumjohann, W., Treumann, R. A., Sckopke, N., and Lühr, H.: Low-frequency waves in the near-Earth's plasma sheet J. Geophys. Res., 100, 9605, 1995.

Borovsky, J. E., Elphic, R. C., Funsten, H. O., and Thomsen, M. F.: The Earth's plasma sheet as a laboratory for flow turbulence in high- $\beta$ MHD, J. Plasma Phys., 57, 1, 1997.

Büchner, J. and Zelenyi, M. F.: Deterministic chaos in the dynamics of charged particles near a magnetic field reversal, Phys. Lett. A 118, 395, 1986.

Büchner, J. and Zelenyi, L. M.: Regular and chaotic charged particle motion in magnetotaillike field reversals, 1. Basic theory of trapped motion, J. Geophys. Res., 94, 11 821, 1989.

Daughton, W.: Kinetic theory of the drift kink instability in a current sheet, J. Geophys. Res., 103, $29429,1998$.

Daughton, W.: Two-fluid theory of the drift kink instability, J. Geophys. Res., 104, 28 701, 1999.

Delcourt, D. C. and Belmont, G.: Ion dynamics at the earthward termination of the magnetotail current sheet, J. Geophys. Res., 103, 4605, 1998.

Greco, A., Taktakishvili, A. L., Zimbardo, G., Veltri, P., and Zelenyi, L. M.: Ion dynamics in the near Earth magnetotail: magnetic turbulence versus normal component of the average magnetic field, J. Geophys. Res., 107, 115, 2002.

Hesse, M., Winske, D., and Kuznetsova, M. M.: Hybrid simulation of collisionless tearing, J. Geophys. Res., 100, 18 929, 1995.

Hesse, M., Winske, D., Kuznetsova, M. M., Birn, J., and Schindler, K.: Hybrid modeling of the formation of thin current sheets in magnetotail configurations, J. Geomagn. Geoelectr., 48, 749, 1996.

Hoshino, M., Nishida, A., Yamamoto, T., and Kokubun, S.: Turbulent magnetic field in the distant magnetotail: Bottom-up process of plasmoid formation?, Geophys. Res. Lett., 21, 2935, 1994.

Hoshino, M., Yamamoto, T., Mukai, T., Nishida, A., and Kokubun, S.: Structure of plasma sheet current in distant magnetotail: Doubly humped electric current sheet, Adv. Space Res., 18, 5, 1996.

Lapenta, G. and Brackbill, J. U.: 3-D reconnection due to oblique modes: a simulation of Harris current sheets, Nonlin. Proc. Geophys., 7, 151, 2000.

Lui, A. T. Y. and Najimi, A.-H.: Time-frequency decomposition of signals in a current disruption event, Geophys. Res. Lett., 24, 3157, 1997.

Lui, A. T. Y., Chang, C.-L., Manfofsky, A., Wong, H.-K., and Winske, D.: A cross-field current instability for substorm expansions, J. Geophys. Res., 96, 11 389, 1991.

Malara F., Veltri, P., and Carbone, V.: Competition among nonlinear effects in tearing instability saturation., Phys. Fluids B, 4, 3070, 1992.

Matthaeus, W. H., Ambrosiano, J. J., and Goldstein, M. L.: Particle acceleration by turbulent magnetohydrodynamic reconnection, Phys. Rev. Lett., 53, 1449, 1984.
Nakamura, R., Baumjohann, W., Runov, A., Volwerk, M., Zhang, T. L., Klecker, B., Bogdanova, Y., Roux, A., Balogh, A., Réme, H., Sauvaud, J. A., and Frey, H. U.: Fast flow during current sheet thinning, Geophys. Res. Lett., 29, 55, 2002.

Ohtani, S., Higuchi, T., Lui, A. T. Y., and Takahashi, K.: Magnetic fluctuations associated with tail current disruption: fractal analysis, J. Geophys. Res., 100, 19 135, 1995.

Peroomian, V., Ashour-Abdalla, M., Zelenyi, L. M., and Petrukovich, A. A.: Intrinsic self-adjustment and variability of the magnetotail, Proceedings of the Fifth International Conference on Substorms, St. Petersburg, 16-20 May, ESA SP-443, 121-126, 2000.

Petrukovich, A. A.: The growth phase: comparison of small and large substorms, Proceedings of the Fifth International Conference on Substorms, St. Petersburg, 16-20 May, ESA SP-443, 914, 2000.

Pritchett, P. L. and Büchner, J.: Collisionless reconnection in configurations with a minimum in the equatorial magnetic field and with magnetic shear, J. Geophys. Res., 100, 3601, 1995.

Runov, A., Nakamura, R., Baumjohann, W., et al.: Cluster observation of a bifurcated current sheet, Geophys. Res. Lett., 30, pp 8-1, doi: 10.1029/2002GL016136, 2003.

Sergeev, V. A., Mitchell, D. G., Russel, C. T., and Williams, D. J.: Structure of the tail plasma/current sheet at $\sim 11 R_{E}$ and its changes in the course of a substorm, J. Geophys. Res., 98, $17345,1993$.

Sergeev, V. A., Runov, A., Baumjohann, W., et al.: Current sheet flapping motion and structure observed by Cluster, Geophys. Res. Lett., 30, pp 60-1, doi:10.1029/2002GL016500, 2003.

Slavin, J. A., Smith, E. J., Sibeck, D. G., Baker, D. N., Zwickl, R. D., and Akasofu, S.-I.: An ISEE 3 study of average and substorm conditions in the distant magnetotail, J. Geophys. Res., 90, $10875,1985$.

Veltri, P., Zimbardo, G., Taktakishvili, A. L. and Zelenyi, L. M.: Effect of Magnetic Turbulence on the Ion Dynamics in the Distant Magnetotail, J. Geophys. Res., 103, 14 897, 1998.

Wiegelmann, T. and Büchner, J.: Kinetic simulations of the coupling between current instabilities and reconnection in thin current sheets, Nonlin. Proc. Geophys., 7, 141, 2000.

Zelenyi, L. M., Milovanov, A. V., and Zimbardo, G.: Multiscale magnetic structure of the distant tail: self-consistent fractalapproach, in: New Perspectives on the Earth's magnetotail, edited by Nishida, A., Baker, D. N., and Cowley, S. W. H., Geophys. Mon. Ser. 105, AGU, 321, 1998.

Zelenyi, L. M., Sitnov, M. I., Malova, H. V., and Sharma, A. S.: Thin and superthin ion current sheets. Quasi-adiabatic and nonadiabatic models, Nonlin. Proc. Geophys., 7, 127, 2000.

Zimbardo, G., Veltri, P., Basile, G., and Principato, S.: Anomalous diffusion and Lévy random walk of magnetic field lines in three dimensional turbulence, Phys. Plasmas, 2, 2653, 1995.

Zimbardo, G., Greco, A., Veltri, P., Taktakishvili, A. L., Milovanov, A. V., and Zelenyi, L. M.: Ion dynamics in the pre-substorm phase: influence of magnetic turbulence and of the normal component of the magnetic field, in Proceedings of the Fifth International Conference on Substorms, St. Petersburg, 16-20 May, ESA SP-443, 225-228, 2000. 